\title{
9 „Ein toter Sartre ist ein guter Sartre“. Bilanz und Ausblick
}

Solang er lebt, ist der Mensch „der potentielle Mensch“"1, der sich erneuernde Mensch. Von Identität könne jedenfalls keine Rede sein, legt Michael Scharang ganz auf Sartres Linie fest: „Nur im Tod stimmt das Ich vollkommen mit sich überein. Denn das lebende Ich bewegt sich mit jeder Gefühlsregung, mit jedem Gedanken, mit jeder Handlung von sich weg.“2 Am Ende bleibt vom Ich dann ein Bündel von Erlebnissen, die Gesamtheit seiner Taten (,’'unité de ses conduites“3), die auszulegen Sache der Lebenden ist, zu denen das Ich nicht mehr gehört. Posthum den Anderen überantwortet - Sartre thematisiert diese Vereinnahmung vor allem im Drama Huis clos - besteht keine Sicherheit, dass die Hinterbliebenen im Sinne der Verblichenen werten und handeln. Sie tun es bei Sartre selbst jedenfalls nicht, klagt Günther Nenning im FORVM mit Blick auf den ,gewaltigen Leichenzug“ auf den Pariser Boulevards, eine „unpassend etablierte Show“: „Gewissenhaft bringen sie den großen Mann zum zweitenmal um, durch Überschütten mit Lob. Ein toter Sartre ist ein guter Sartre.“4 Was nach Sartres Tod am 15. April 1980 in der österreichischen Presse geschieht, lässt sich zwar nur eingeschränkt als Überschütten mit Lob bezeichnen, doch aber als Geste des Vergebens. Vergeben werden die Jahre, in denen er in die Irre gegangen ist, gerade in Wien.

Kaum ein Nachruf, gleich aus welchem Teil des politischen Spektrums, lässt diese Fehltritte unerwähnt. „Seine Widersprüchlichkeit darf uns seine Größe nicht verkennen lassen“, mahnt in der prokommunistischen Volksstimme Arthur West, der an Sartre die „mutigen gesellschaftlichen Engagements“ als „von dauerndem Wert“ lobt und der „überragenden Persönlichkeit“ nur vorhält, sich zuletzt wieder „in antisowjetische Positionen“5 verstrickt zu haben. Die bürgerlich-liberale Presse nimmt auf Seite eins Abschied vom „Leitfossil“, das sich, unbeeindruckt von „Erfolg, Kritik oder auch materielle[m] Wohlstand“, nie scheute, „Irrtümer zuzugeben oder die Rolle eines politischen Harlekins vornehmlich der linken Szene zu spielen“6. Kurt Wimmer von der Kleinen Zeitung

1 Musil: Der Mann ohne Eigenschaften, S. 251.

2 Michael Scharang: Das Geschwätz von der Identität (1992). In: Scharang: Bleibt Peymann in Wien oder kommt der Kommunismus wieder, S. 114-124, hier S. 120.

3 Sartre: À Propos de l'existentialisme, S. 654.

4 Nenning: Sankt Sartre, S. 20, 21.

5 Arthur West: In memoriam Jean-Paul Sartre. In: Volksstimme, 17.04.1980.

6 ile: Ein Leitfossil. In: Die Presse, 17.04.1980.

Ә Open Access. (c) 2021 Juliane Werner, publiziert von Walter de Gruyter. (c) BY Dieses Werk ist lizensiert unter einer Creative Commons Namensnennung 4.0 International Lizenz. 
verliert derweil den Überblick: „Er hat sich selbst so oft kritisiert, dementiert und überholt, daß es gar nicht leicht ist, in wenigen Zeilen zu sagen, was dieser JeanPaul Sartre wirklich war."7 Insofern als Veränderung zu den Konstanten des existentialistischen Denkens zählt, wagt Franz Mayrhofer in den Salzburger Nachrichten das Fazit, dass sich Sartre ,in den wesentlichen Zügen treu geblieben“8 ist. Franz Schuh äußert in seinem „Lest Sartre!“ betitelten Nachruf im Profil ebenfalls: „Sartre war, sosehr auch das Gegenteil behauptet wird, niemals ein Richtungsphilosoph. "9 Hans Heinz Hahnl, der Sartres Entwicklung in der sozialdemokratischen Arbeiter-Zeitung lang verfolgt, sieht einen unabhängigen „Mann zwischen den Fronten, oft genug zwischen allen Sesseln, der sich weder vom Westen noch vom Osten institutionalisieren lassen wollte“:

\begin{abstract}
Sowohl in der Geistesgeschichte wie in der politischen Geschichte Nachkriegseuropas ist er als einer der wichtigsten Anreger, Denker, Aktivisten eingegangen. Er hat sich nicht mit dem Wort begnügt, er hat sein Wort immer für das eingesetzt, was er für richtig gefunden hat, ohne Rücksicht auf Ideologien und politische Konstellationen. ${ }^{10}$
\end{abstract}

Der beste Beweis für Sartres Gewicht liegt Peter Kampits zufolge darin, „daß sein Abwesendsein auch jene bestürzt, die seinem Denken und Handeln die Gefolgschaft verweigerten“11. Nichtsdestotrotz gibt es durchaus auch weniger wohlwollende Bilanzen: So scheint man bei der oberösterreichischen christlichsozialen Tageszeitung Neues Volksblatt noch immer aufzuatmen, dass Sartres „Philosophie, die sich einst wie eine ,Epidemie‘ verbreitet hatte, heute passé ist“"12. Zu einem ernüchternden Ergebnis, was den späten Sartre angeht, kommt Rudolf John im Kurier: „Er war in den letzten Jahren unmodern geworden, vergessen fast, verdrängt aus der Szene jungen Denkens, als dessen Idol er lange Zeit figurierte. “13 Würdigt der Wiener Kulturkritiker Friedrich Heer am 16. April 1980 in einem Radio-Gespräch „diesen lebenslang jugendlichen, jugendbewegten Menschen Sartre“14, gibt ein Text aus seinem Nachlass auf die Frage „Wie viele Jugenden und ihr zugehöriges Scheitern hat Sartre erlebt?“ die eher nega-

7 Kurt Wimmer: Der alte Mann und der Tod. In: Kleine Zeitung, 17.04.1980.

8 Franz Mayrhofer: Sartre: Tod mit der Hoffnung. In: Salzburger Nachrichten, 17.04.1980.

9 Schuh: Lest Sartre! In: Profil, 21.04.1980.

10 Hans Heinz Hahnl: Der Mann zwischen den Fronten. In: Arbeiter-Zeitung, 17.04.1980.

11 Peter Kampits: In Freiheit scheitern. In: Die Furche, 23.04.1980.

12 o. V.: Der Mensch zu Freiheit verurteilt. In: Neues Volksblatt, 17.04.1980.

13 John: Nur Sprache und Denken. In: Kurier, 17.04.1980.

14 Friedrich Heer (im Gespräch mit Konrad Zobel): Ö1-Mittagsjournal vom 16.04.1980, 12:12:0012:18:46; Österreichische Mediathek. https://www.mediathek.at/atom/08F8A74B-2A0-000DC00000EFC-08F80863 (einges. 09.01.2019). 
tive Antwort, der Autor sei ein „grosses Kind“, das, etwa bei der Absage an den Stalinismus, „nicht der erste, sondern immer der letzte sein“ wolle:

Die Fremdheit, die den späten Sartre umgibt, als er, längst eine Reliquie, ein „Grosspapa“, ein „Mann von Gestern“ geworden, auf die Strasse geht, um an Demonstrationen einer Jugend teilzunehmen, die unendlich fern vom Aufstand des Knaben Sartre in seiner ersten Jugend leben [!], kann beklemmend wirken. ${ }^{15}$

Was also bleibt?, überlegt Rudolf John und kommt zu dem Schluss, nicht viel: Sartres Philosophie - „Grundlage für alle weiteren philosophischen Denkvorstöße in diesem Jahrhundert“ und dennoch „zu quälend [...], um in Mode zu bleiben“ - habe nun ihren „Platz im staubigen Museum der aufgegebenen Erkenntnisse“; aber zumindest seine Prosa (zu denken wäre hier an La Nausée) sichere ihm „einen festen Platz in den Lexika aller Zeiten“16. Ein Platz sicher ist dort auch Albert Camus, nach dessen tödlichem Autounfall am 4. Jänner 1960 die österreichischen Periodika uneingeschränkt positiv - und, fast ausnahmslos, ohne den Nicht-mehr-Freund Sartre zu erwähnen - berichten. Die Tode beider Autoren lösen eine Rückkehr ihrer Dramen in die österreichischen Spielpläne aus, 1980 erscheinen Friedrich Heer im Ö1-Radiointerview Sartres Stücke keineswegs als in die Jahre gekommen:

Es wär' übrigens an der Zeit, wirklich jetzt wieder einige seiner bedeutenden Stücke [zu spielen], die Sachen sind sehr aktuell, nicht? Man könnte, also wenn ich etwas zu sagen hätte, würde ich heute für junge Generationen, junge Generationen zwischen 20 und 70 Jahren, Sartre aufschlüsseln, indem ich Stücke von ihm bringen würde, Dramen, die zeigen, dass ja alles das, was er an oft nackter Verzweiflung erlebt hat, nicht, ja doch noch Gegenwart ist, nicht, die Sache des Menschen steht heut', steht 1980 nicht besser als $1940 .^{17}$

Als sich im Folgejahrzehnt die Beliebtheit der Sartreschen Bühnenwerke wieder abschwächt, ist es Claus Peymann, der als scheidender Burgtheaterdirektor auf ihre Aktualität hinweist:

Was jetzt vielleicht fällig wäre, wenn ich hierbleiben würde, hat mir interessanterweise ein Wiener Taxifahrer gesagt. Der ließ sich auf gar nichts ein, fragte nur: Wieso spielen Sie eigentlich nicht Sartre und Camus? Das sind doch Autoren, in denen sich eine politische Bewältigung unserer Gegenwart findet. Der Mann hat recht. Der Wiener Taxifahrer hat recht. ${ }^{18}$

15 Heer: In memoriam Jean Paul Sartre (LIT), S. 1, 5, 1.

16 John: Nur Sprache und Denken. In: Kurier, 17.04.1980.

17 Heer: Ö1-Mittagsjournal vom 16.04.1980, 12:12:00-12:18:46.

18 Claus Peymann: Peymanns Abrechnung. In: Profil, 21.12.1998. 
Bevor das Revival des Existentialismus nach Sartres Ableben wieder vergehen kann, veranlasst der politische Rechtsruck Mitte der achtziger Jahre einen neuen, vielfach von Sartre inspirierten Zusammenschluss von Literatur und Politik, im Zuge dessen intellektuelles Intervenieren und schriftstellerisches Engagement eine erhebliche Aufwertung erfahren. Das aufdeckende Schreiben begleitet nun, anders als in den frühen sechziger Jahren, eine öffentliche Debatte über Österreichs einstige und seinerzeitige Haltung zum Nationalsozialismus. Dabei wird der Opferstatus, auf dessen Basis die französischen Alliierten nach 1945 eine mit dem Existentialismus-Transfer verwobene Schulddiskussion wie in der Bundesrepublik Deutschland vermeiden, zuletzt delegitimiert.

Abgesehen von der enthüllenden littérature engagée reicht die Präsenz des Existentialismus in Texten österreichischer AutorInnen von der Behandlung analoger existenzphilosophischer Themen und Motive (z. B. bei Dor, Aichinger, Bachmann und Lebert) über namentliches Aufrufen (z. B. bei Blaha, Fritsch, Simmel und Turrini) bis zu markierten Zitaten und eingehender Auseinandersetzung mit den Theoremen Sartres und Camus' (z. B. bei Okopenko, Winkler, Jelinek und Gstrein). Dass den SchriftstellerInnen, die explizit auf den Existentialismus verweisen, viele gegenüberstehen, deren Werke ohne solche Nennungen ähnlichen literaturtheoretischen Wegen folgen oder sich inhaltlich mit der französischen Literatur der situations extrêmes überschneiden, verdeutlicht die Macht der zeitgeschichtlichen Umstände, die diese Konvergenzen hervorbringen.

Wiederum andere AutorInnen, etwa aus dem avantgardistischen Umfeld der „Wiener Gruppe“, erwähnen ihre Beschäftigung mit Sartre oder Camus, verfassen aber Bücher, in denen sich der Transferforschungsaufgabe, „das Fremde im Eigenen zu entdecken“19, in Bezug auf Existentialismus-Spuren nur schwer nachkommen lässt. Dass sich diese Einflüsse nicht erkennbar literarisch niederschlagen, liegt auch an ihrem Verschmelzen mit gleichzeitig aufgenommenen, bis dato kriegsbedingt vernachlässigten anderen Strömungen aus dem Ausland, vor allem mit dem Surrealismus. Die internationalen Kulturgüter treffen zudem nach 1945 im Aufnahmekontext mit Neuentdeckungen aus der eigenen Kultur zusammen, vornehmlich von Franz Kafka. Nachdem dieser als Schwergewicht innerhalb der „tradition of literary-philosophical hybrids“ ${ }^{20}$ in den vorangegangenen Jahrzehnten schon Sartre und Camus geprägt hat, verstärken deren Werke nun die Wirkung Kafkas in Österreich. In diesem Aspekt, wie auch in seiner Verankerung in der deutschsprachigen Phänomenologie und Existenz-

19 Jurt: Das wissenschaftliche Paradigma des Kulturtransfers, S. 31.

20 Collins: The Sociology of Philosophies, S. $764 \mathrm{f}$. 
philosophie, zeigt der Existentialismus idealtypisch, wie Kulturtransfers in eine Geschichte aufeinanderfolgender Hybridisierungen eingebunden sind. ${ }^{21}$

Zwar verwandeln sich die Aufnehmenden Kulturgüter nach ihren eigenen Bedürfnissen an, doch ist die VermittlerInnenebene beim ExistentialismusTransfer durch die spezifische Machtdynamik der Besatzungszeit von besonderer Bedeutung. Die Alliierten üben ab 1945 im Kulturbetrieb entscheidende Funktionen in allen Transferkanälen aus, im Buchhandel, in öffentlichen Bibliotheken, in privaten Leihbüchereien, Rundfunk und Wochenschauen, Schulen und Universitäten, bei der Presse und am Theater. Das „Hereinströmen der freiheitsatmenden Weltliteratur“ ${ }^{22}$, auch des Existentialismus, erfolgt dabei primär über Literatur- und Kulturzeitschriften. Maßgeblich beteiligt an diesem Prozess sind zwischen den Kulturen stehende VermittlerInnen, häufig mit Besatzungshintergrund, die durch die Selektion existentialistischer Elemente letztlich gegen die vorgegebene kulturpolitische Linie handeln: Diese sieht zur Stärkung des österreichischen Nationalgefühls den Einsatz von nicht-polarisierender und -beunruhigender Hochkultur vergangener Zeiten vor.

Bevor die Übersetzungen existentialistischer Werke durch die seit 1948 allmählich wieder zugängigen deutschen Verlage in substantiellem Ausmaß eintreffen und die benachbarten Literaturbetriebe einander näherrücken, etwa durch Vernetzung der AutorInnen der „Gruppe 47“, werden die österreichischen Rezeptionspartikularitäten besonders evident. Neben dem Überspringen von Les Mouches - mitbedingt durch das Umgehen von Selbstreflexion auslösenden Maßnahmen seitens der Besatzung - ist dies vor allem die stark katholisch geprägte Aufnahme in einer Zeit, in der sich mithilfe des beliebten Renouveau Catholique die Literatur rekonfessionalisiert. Als christliche ExistentialistInnen aufgefasste Philosophen wie Gabriel Marcel werden durchgehend positiv besprochen, allerdings in weit geringerem Umfang als der Atheist Camus, der in Österreich nicht selten zum ,erfolgreichsten Verbreiter der Philosophie Sartres“،23 umfunktioniert wird, und als Sartre selbst, der durch seine Breitenwirkung als „international celebrity“ ${ }^{24}$ schlecht ignoriert werden kann. Die Abwehrversuche seiner als Pessimismus und Nihilismus empfundenen Lehre durch katholische Organe (die gelegentlich auch Vereinnahmungsbemühungen anstellen) haben wesentlichen Anteil am Transfer, nicht zuletzt, indem sie sie auf diese Weise mit subkulturellem Kapital für die Jugend und für Künstlerkreise aufladen, unter

21 Cf. Espagne: La Notion de transfert culturel, S. 3.

22 Fischer: Um ein geistiges Österreich!, S. 99.

23 Fischl: Idealismus, Realismus und Existentialismus der Gegenwart, S. 319.

24 Baert: The Existentialist Moment, S. 148. 
denen die existentialistischen Ideen (gestützt durch Berichterstattung in geneigten Periodika) am stärksten zirkulieren.

Betreffend Sartres scheinbaren Anti-Humanismus deckt sich die katholische mit der kommunistischen Haltung in den ersten Nachkriegsjahren. Sind in seiner antikommunistischen Phase durchaus noch kommunistische oder dem Kommunismus sehr nahe stehende SchriftstellerInnen in Österreich öffentlich aktiv, gerade im PEN-Umfeld, stellen diese, als Sartre 1952 in Wien öffentlich zum compagnon de route wird, eine Splittergruppe im inzwischen ausdrücklich antikommunistischen Klima dar. Zwischen den Blöcken gelegen, gerät Österreich zum Austragungsort auch von kulturellen Konflikten des Kalten Kriegs der vier alliierten Mächte, so beim berühmten Brecht-Boykott und bei den Skandalen um Sartre in der ersten Hälfte der fünfziger Jahre. ${ }^{25}$ Sein Völkerkongress-Auftritt und der medial befeuerte Kampf um die Wiener Les Mains sales-Aufführungen bilden den Höhe- und Wendepunkt des Transfergeschehens und stellen für die weitere Existentialismus-Aufnahme die Weichen. Auch wenn Sartres Verhältnis zur Kommunistischen Partei schnell wieder ins Negativ(er)e umschlägt, verfestigen diese Momente in Österreich sein Image, Literatur für (partei)politische Zwecke zu missbrauchen und den eigenen Figuren „ideologische Spruchbänder zum Hals heraushängen“'26 zu lassen.

Im Verlauf der fünfziger Jahre, einer Phase allgemeiner politischer und ökonomischer Stabilisierung, verliert der Existentialismus als „Philosophie der Krise“ ${ }^{\text {27 }}$ - geboren aus den kollektiven und individuellen Grenz- und Zäsurerfahrungen des Krieges, der Besetzung und der Libération - an „Durchschlagskraft“"28. Anders als in Frankreich, wo Sartre bei den 1968er-Unruhen als Intellektueller neuerlich in der ersten Reihe steht und durch seine politischen Interventionen im Schulbildungsbereich höchstens Eklats auslöst, ${ }^{29}$ gelten seine existentialistischen Schriften in dem von der Protestbewegung

25 Diese selten auffallende Parallele erwähnt anlässlich der Burgtheater-Premiere von Sartres Die Fliegen im Jahr 1965 Ernst Lothar (Tragikomödie der Gewissensbisse. In: Express, 16.02.1965): „Eine Frage: Wird auf dem Lueger-Ring mit ungleichem Maß gemessen? Da man dort Brecht mit dem Bann belegt, was rechtfertigt das Privileg zugunsten des ihm ideologisch nicht fernstehenden Sartre? Sein deprimierendes Résistancestück, das man übrigens in Wien schon sah, keinesfalls.“

26 Basil: Jean Paul Sartre, Die schmutzigen Hände, Theater in der Josefstadt, S. 267.

27 Fischl: Idealismus, Realismus und Existentialismus der Gegenwart, S. 236.

28 Jurt: Jean-Paul Sartre oder der totale Intellektuelle. In: Neue Zürcher Zeitung, 22.05.1987. Cf. Bourdieu: Les Règles de l'art, S. 350.

29 Cf. Jean-Paul Sartre: „Le Mur“ au lycée. In: Sartre: Situations, VIII, S. 233-238. [Zuerst in: Le Monde, 18.01.1969.] 
weitgehend unberührten Österreich jüngeren LehrerInnen inzwischen als harmlos genug für die Verwendung als Lehrmaterial: Zusammen mit Camus' Literatur werden sie zu Klassikern im Schulunterricht und damit auch schon bald zur geistesverändernden Lektüre für zahlreiche angehende SchriftstellerInnen. ${ }^{30}$

Als Epochenetikett laut Jürgen Link „,in der Regel primär philosophiegeschichtlich definiert“31, bleibt dem Existentialismus in Österreich der Status einer ernstzunehmenden Philosophie aufgrund seines Naheverhältnisses zu Literatur und Politik sowie durch seine Ausdrucksformen als Lebensstil zunächst verwehrt. Nachdem diese „philosophische Modeströmung in breiten Schichten

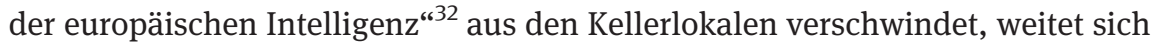
die universitäre Befassung mit seinen Erzeugnissen langsam aus. Zuvor wird in wissenschaftlichen Publikationen - im Ton ähnlich der katholischen Kulturkritik, mit der es zahlreiche personelle Überschneidungen gibt - vor allem Sartre wortreich blockiert. Mit Ausnahme weniger, aber durchaus wichtiger Lehrveranstaltungen und Hochschulschriften in der Zeit der Erstaufnahme, etabliert sich Sartre ebenso wie Beauvoir, Camus, Marcel und Merleau-Ponty als VertreterInnen (und ScheinvertreterInnen) des Existentialismus akademisch erst in den 1970er Jahren.

Dass Sartre nach dem Zweiten Weltkrieg auch in Österreich „eine der Gali-

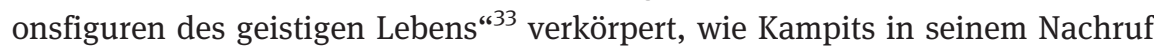
formuliert, lässt sich auf allen Ebenen des geistigen Lebens - wenn auch teils für überschaubare RezipientInnengruppen - belegen. Seinen Nachhall zu ermessen, ein im 21. Jahrhundert andauerndes Projekt, ${ }^{34}$ fühlt man sich 1980 bei der Presse indes noch nicht bereit: „Welche Wirkung sein Leben und sein Werk auf zwei Generationen der Intelligenz Europas, aber ebenso Japans, Afrikas und Amerikas hatten, ist auch heute noch nicht abzusehen." ${ }^{\text {35 }}$ Sartre selbst ist

30 Eine pessimistische Prognose zu einer den Existentialismus umfassenden gegenwärtigen Schullektüre äußert Ernst Wirthensohn: Wann wehren wir uns endlich! In: Die Presse, 07.02.2015: „Die Tatsache, dass Fremdsprachen vor allem über literarische Texte vertieft werden können, scheint keine Relevanz mehr zu haben. Die Folge: Wurden noch vor einigen Jahren sehr häufig Werke englischer und auch französischer Literatur ausgeliehen, so verstauben nun diese Regale; Entlehnungen sind eine Seltenheit geworden, die Maturanten kennen keine Namen mehr wie Dickens, Austen, Woolf, Beckett, Steinbeck oder Flaubert, Zola, Camus, Beauvoir und Sartre.“

31 Link: Denormalisierung als Grenzsituation, S. 265.

32 Benedek: Zweimal Jean Paul Sartre. In: Tagebuch, 03.01.1953.

33 Kampits: In Freiheit scheitern. In: Die Furche, 23.04.1980.

34 Cf. etwa die Bände Situating Existentialism (2012) von Judaken und Bernasconi sowie Sartre and the International Impact of Existentialism (2020) von Betschart und Werner.

35 ile: Ein Leitfossil. In: Die Presse, 17.04.1980. 
an seinem Nachruhm wenig interessiert und nimmt durch seine Weigerung, eine überzeitlichen Idealen verpflichtete Literatur zu verfassen, ein mögliches Schwinden seines Lesepublikums in Kauf. Auf die Wirkung seiner Werke in situ legt er allerdings großen Wert, auch in Österreich, wie er 1952 der Presse mitteilt: „Es bedeutet für mich viel, daß man sich in den westeuropäischen Ländern, namentlich in Deutschland, in der Schweiz, in Österreich, mit meinem Schreiben befaßt und ihm Gerechtigkeit widerfahren läßt.،“36

36 Jean-Paul Sartre. In: S. F.: Besuch bei Jean-Paul Sartre. In: Die Presse, 12.07.1952. 
